\title{
An Adaptive PID Speed Controller for an 8/6 Switched Reluctance Machine
}

\author{
Silviano Rafael \\ Dep. of Electrical Engineering \\ Polytechnic Institute of Setubal \\ IPS/ESTSetúbal \\ Setúbal, Portugal \\ silviano.rafael@estsetubal.ips.pt
}

\author{
P.J.Santos \\ Dep. of Electrical Engineering- \\ Polytechnic Institute of Setubal \\ IPS/ESTSetúbal/INESC \\ Setúbal, Portugal \\ paulo.santos@estsetubal.ips.pt
}

\author{
A.J. Pires \\ Dep. of Electrical Engineering- \\ ESTSetúbal/IPS \\ CTS-UNINOVA \\ Portugal \\ armando.pires@estsetubal.ips.pt
}

\begin{abstract}
This paper presents a classical controller with parameters adaptation capability, in an automatic way. This controller is based on a PID where a parameters adaptation algorithm is used and applied to the switched reluctance motor (SRM) speed control. This PID design do not require any kind of adjustment or calibration from the operator. The parameters adaptation algorithm implemented is based on one fuzzy system with a Takagi-Sugeno inference mechanism with some simplifications. These simplifications had the goal to select the parameters adaptation algorithm contributing for a fast controller response. The developed adaptive PID algorithm was modelled and simulated.
\end{abstract}

Keywords- Switched reluctance motor; Speed control; PID control; Adaptative parameters system; Fuzzy Takagi-sugeno.

\section{INTRODUCTION}

The PID controller is heavily used in the control loops of industrial processes but its parameters need to be tuned for a smooth operation. These parameters are dependent of the control process variables that remain unchanged during its regular activity. The start up of the PID controller is not always a simple work in the parameters adjustment, besides the existence of some methodologies [1]. Despite the helpful of these methodologies in the approached parameters values calculation, is however necessary an observation period to survey with greater certainty the controller performance, which requires, in some cases, a substantial amount of time.

In the classical speed control of electrical machines it is usual to have one linear PID controller. This kind of controller can present a good result near one operating point, where the machine can be considered as a linear system. However, it has some limitations if it is planed to be used in a wide working region. More complex cases exist due to their particularities, where the system under control is non linear, like the switched reluctance motor (SRM) that compromise the PID controller performance.
These two problems above referred, tuning parameters and the behaviour of the controller, are interconnected and attracted the attention of many researchers. In the literature, some works, to solve these drawbacks, have been proposed. These drawbacks are difficult to solve with conventional control techniques due to the complexity of modeling the SRM dynamics. Some examples are the application of variable structure controller [2], "intelligent" controllers using fuzzy logic and neural networks $[3,4]$ and hybrid system [5, 6 and 7]. Several of these proposals require high processing power to perform the necessary calculation matrix which demand a more expensive controller's hardware.

One way to solve this problem is based on the gain scheduling technique. However some know how of the process is needed to define the gain matrix to schedule it for each SRM functioning regimen.

Another way is based on the adaptive gain of the PID speed controller and it is presented in this paper. This proposal contributes to reduce or even to prevent the already referred problems. For this purpose, basically, it is necessary to understand the mathematical model of SRM (section II), to present the adaptive controller design (section III and IV) and to present the simulation results and analyze the behaviour of the speed control system (section V).

\section{MATHEMATICAL MODEL OF SRM}

The SRM model is considered complex due to its magnetic circuit non linearity. The SRM electrical equations of one phase can be expressed as:

$V_{k}=R i_{k}+\frac{\partial \lambda_{k}\left(\theta, i_{k}\right)}{\partial t}$ 
where the influence of the mutual flux was neglected and $V_{k}$ is the phase voltage, $i_{k}$ is the phase current, $R$ is the resistance per phase, $k$ is the active phase and $\lambda_{k}\left(\theta, i_{k}\right)$ is the phase linkage flux. Using the chain rule, (1) can be rewritten as:

$$
V_{k}=R i_{k}+\frac{\partial \lambda_{k}\left(\theta, i_{k}\right)}{\partial i_{k}} \frac{d i_{k}}{d t}+\frac{\partial \lambda_{k}\left(\theta, i_{k}\right)}{\partial \theta} \frac{d \theta}{d t}
$$

The torque developed by one excited phase is determined by the variation of the magnetic co-energy produced in its magnetic circuit in order to the variation of the position and it is expressed in (3).

$$
T\left(\theta, i_{1}, \ldots, i_{n}\right)=\left.\frac{\partial W^{\prime}\left(\theta, i_{1}, \ldots, i_{n}\right)}{\partial \theta}\right|_{i_{1}, \ldots, i_{n}=\text { const }}
$$

The magnetic co-energy is characterized by the following expression (4).

$$
W^{\prime}\left(\theta, i_{1}, \ldots, i_{n}\right)=\left.\sum_{k=1}^{n} \int_{0}^{i_{k}} \lambda_{k}\left(\theta, i_{k}\right) d i_{k}\right|_{\theta=\text { const }}
$$

\section{THE PROPOSED SPEED CONTROLLER}

The block diagram of the proposed speed controller system with the converter and the 8/6 switched reluctance motor is presented in Fig 1. The used PID controller with all the function block delimited by the dashed square, is in discrete time domain with a sample time of $200 \mu \mathrm{s}$. This PID is characterized by the following equation (5).

$$
u_{k}=u_{k-1}+k p_{k} e_{k}+k i_{k} \sum e_{k}+k d \frac{e_{k}-e_{k-1}}{T s}
$$

Where $e_{k}$ and $e_{k-1}$ are the speed error of the system response in the $k$ and $k-1$ instant, respectively. The parameters of the controller are $k p_{k}, k i_{k}$ and are modified by an algorithm which is represented by the adaptation block in figure 1 . The parameter $k d$ is unitary and was kept constant. The output of the PID algorithm is given by $u_{k}$, and it is used in different ways by the two next blocks. The first block $\left(\left|\mathrm{U}_{\mathrm{k}}\right|\right)$, converts the PID output in absolute values which is used as signal reference for the duty cycle output in the PWM block. The $\left|\mathrm{U}_{\mathrm{k}}\right|$ block is implemented with two saturation values due the limitation of the PWM. The second one gives the signal of $u_{k}$ and it is used to select a sequence of phases (phase's sequence block). In other words the magnitude of $u_{k}$ is, indirectly, the pretended torque value and the signal of $u_{k}$ is to determine the signal of the electromagnetic torque developed on the machine. The signal of the torque is obtained by the selection of the phases sequence presented in table I.

TABLE I

TORQUE SIGNAL PRODUCED AND RESPECTIVE PHASES TO EXCITE

\begin{tabular}{|c|c|c|c|c|}
\hline Angle $\left(^{\circ}\right)$ & $0^{\circ}$ to $15^{\circ}$ & $15^{\circ}$ to $30^{\circ}$ & $30^{\circ}$ to $45^{\circ}$ & $45^{\circ}$ to $60^{\circ}$ \\
\hline Positive Torque & Phase 4 & Phase 3 & Phase 2 & Phase 1 \\
\hline Negative Torque & Phase 1 & Phase 4 & Phase 3 & Phase 2 \\
\hline
\end{tabular}

\section{ADAPTIVE ALGORITHM}

The considered adaptive algorithm intends to have the advantage of simplicity and to be implemented with few processing resources when compared with "intelligent" or hybrid systems. The question related with the processing time is very important because it limits the quickness of the control signal, the quickness of the controller parameters adaptation and consequently it limits the set performance and behaviour in the reference signal tracking.

The adaptive algorithm is inspired in a Tagaki-Sugeno fuzzy system [8], with some simplifications.

In this type of fuzzy system, the condition part uses linguistic variables and the conclusion part is represented by a mathematical function. The proposed system has four conditions and two distinct conclusions and it is characterized by one universe of discourse, which is the speed error percentage, $P e_{k}$. The $P e_{k}$ is defined by (6), where $w_{\text {refk }}$ is the speed reference and $w_{k}$ is the speed in the $k$ instant.

$$
P e=\frac{w_{r e f k}-w_{k}}{w_{r e f k}} * 100
$$

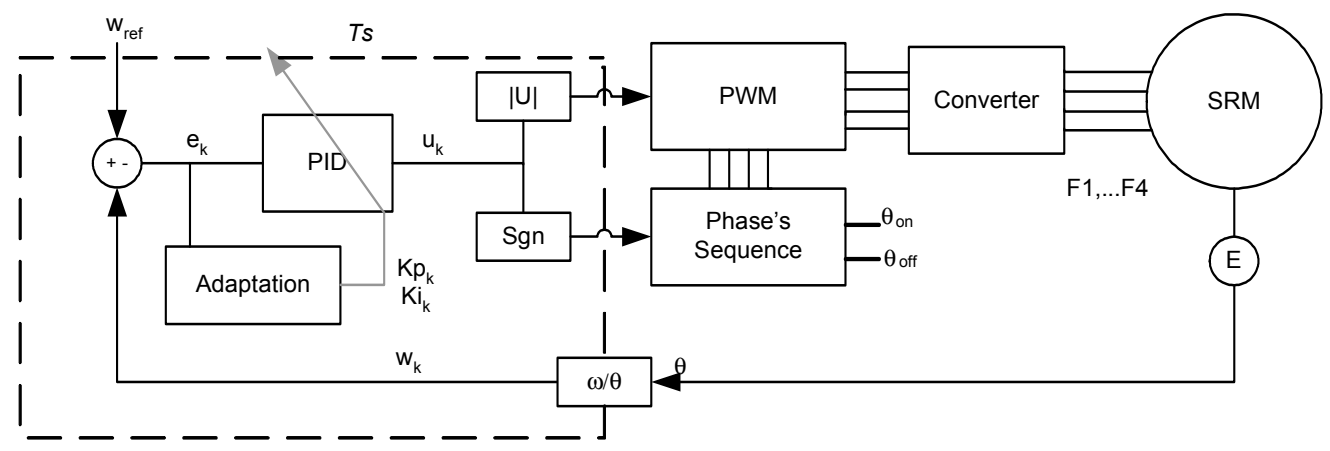

Figure 1 - Block diagram of the SRM speed control system 
The distribution of the four rectangular membership function of the fuzzy system proposed is shown in figure 2 .

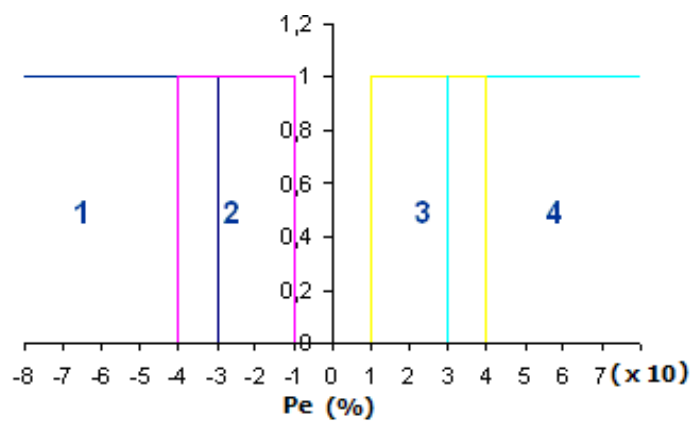

Figure 2 - Distribution and type of membership functions

The membership functions $\mathrm{n}=\{1,4\}$ activate the consequent part given by the expression (7). When the error percentage is lower than -40 or greater than 40 the $K p_{k}$ parameter value is actualized.

$$
K p_{k}=K p_{k-1}+\left(K_{1} e_{k}+\frac{e_{k}-e_{k-1}}{T s}\right) \cdot \vartheta \cdot \mu^{n}
$$

The membership functions $\mathrm{n}=\{2,3\}$ activate the consequent part given by the expression (8). When the error percentage is in the interval ]-30, -10 ] or [ 10, 30[ the $K i_{k}$ parameter value is actualized.

$$
K i_{k}=K i_{k-1}+\left(K_{2} e_{k}+\frac{e_{k}-e_{k-1}}{T s}\right) \cdot \vartheta \cdot \mu^{n}
$$

In expressions (7) and (8) $e_{k}$ and $e_{k-1}$ are the error values in the instant $k$ and $k-1$, respectively. $\mu^{n}$ is the strength activation of the adaptation function of the membership function $n$. The dynamics of the adaptation parameter is controlled by the constants $K_{1}$ and $K_{2}$ that change the relation between the error value in the instant $k$ and its derivative term. The proportionality factor $\vartheta$ is loaded initially with a value between 0 and 1 and will remain constant during the system functioning. The value of this factor is important because it will influence the range of the adaptation parameter.

The error value in the instant $k$ is defined by (9).

$$
e_{k}=w_{r e f k}-w_{k}
$$

Depending on the activation of the membership function in the instant $k$ and the dynamics of the error signal value, the parameters values $K p_{k}$ or $K i_{k}$ can be increased, decreased or stabilized.

The strength activation, $\mu^{n}$, of the membership function assumes the value 0 or 1 due to the use of rectangular membership function and this implies the operation of the adaptation algorithm (7) and/or (8) in order to maintain or change the parameters values.

\section{SimUlation RESUlts}

This controller, with parameter adaptation, was simulated in Matlab Simulink environment. All the models shown in the block diagram in figure 1 were developed. The SRM model is not linear and is based on lookup tables functions with the static torque characteristic and electromagnetic characteristic. These characteristics were obtained experimentally and have been tested and applied in several studies developed by the authors [9, 10 and 11]. The converter model is based on the two power switches topology per SRM phase and the command developed in the phase's sequence block is prepared to apply the functionality of the four quadrant operation. The block $\theta / \mathrm{w}$ converts the position information given by the encoder in speed value. This output block is time sampled dependent. The goal is to provide the type and form of the signal that usually the digital signal controllers for motor control deal.

The simulation results of the system, when it was imposed a rectangular signal of speed reference, are presented in figure 3. The reference speed has two levels, one at 300 RPM and another at 1200 RPM. In figure 3 it is presented the behaviour of the system, where on the third adaptation cycle no overshoot is verified.

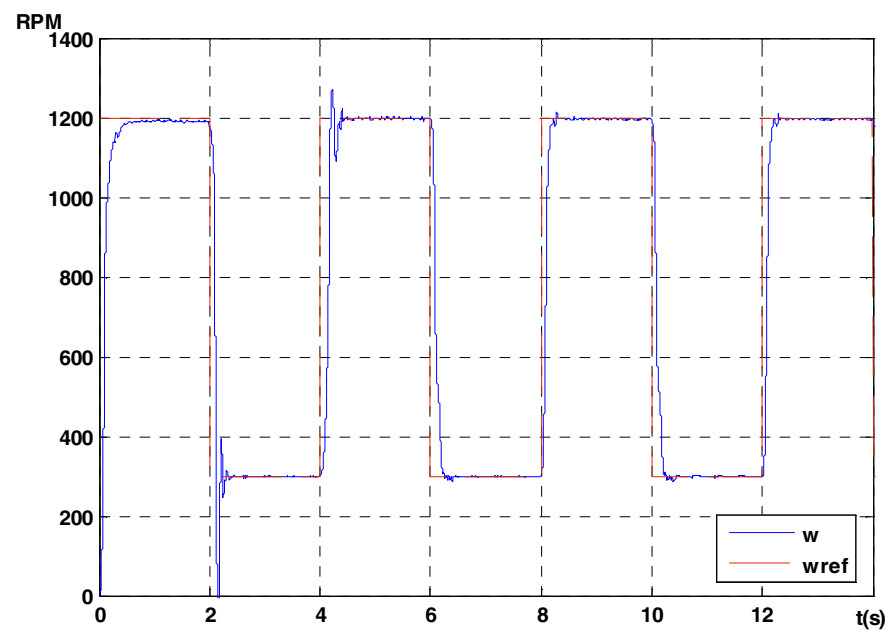

Figure 3 Speed response of the system to reference signal..

The error analysis on the third cycle of the $300 \mathrm{rpm}$ and $1200 \mathrm{rpm}$ steady state is done through the MPE and the MAPE. It consists in the calculation of the error percentage average (MPE - mean percentage error) and the average of the absolute values of the error percentage (MAPE- mean absolute percentage error) expressed in (10) and (11), respectively.

$$
M P E=\sum_{i=1}^{n} \frac{P e_{i}}{n} ; i \in \mathrm{N}^{+}
$$




$$
\text { MAPE }=\sum_{i=1}^{n} \frac{\left|P e_{i}\right|}{n} ; i \in \mathrm{N}^{+}
$$

The calculated values are presented in table 2, to highlight the effectiveness of the adaptation algorithm. It is evident that the performance presented for the $1200 \mathrm{rpm}$ steady state is the best.

Table 2- Speed Error Calculation

\begin{tabular}{|c|c|c|}
\hline $\begin{array}{c}\text { Third cycle } \\
(\text { RPM })\end{array}$ & MAPE(\%) & MPE(\%) \\
\hline 300 & 0,556 & 0,487 \\
\hline 1200 & 0,295 & 0,278 \\
\hline
\end{tabular}

It is also presented in figure 4 the evolution of the PID parameters $K p_{k}$ and $K i_{k}$ registered in the output of the adaptation block, for the case of figure 3 .

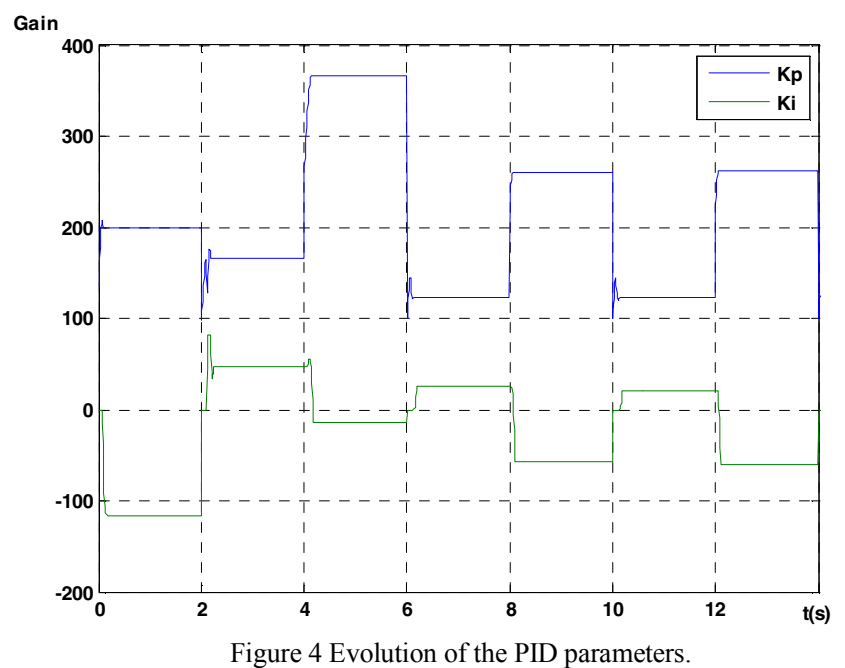

\section{CONCLUSIONS}

This work presents a PID controller with a parameter adaptive algorithm and its performance on a four phases switched reluctance motor (SRM) speed control system..

The controller has one input, based on the speed machine and two outputs, the signal of the torque and the duty cycle value. For the parameters adaptation one fuzzy system with a Takagi-Sugeno inference mechanism was chosen and some simplification of this algorithm system was implemented and verified in the simulation.

The main advantage of the presented system is that it does not need any kind of adjustment or PID calibration. It has the advantage of the adaptive systems, quickly compensating the disturbances that can appear in the system control functioning.

The major drawback is the adaptation dynamic, which must be adjusted through $K_{1}$ e $K_{2}$ and remains unchanged.
The adaptive algorithm that is demonstrated in this work is quite simple, robust and converges quickly. The limitation of the memberships are in $>+30 \%$ and $<-30 \%$ for the $K p_{k}$ and $[+40 \%,+10 \%]$ to $[-10 \%,-40 \%]$ for the $K i_{k}$ values. These memberships' limits values had given the best simulation response results. It is observed that the PI parameters depend on the evolution of the error value and the adaptation factor value. It was used two kinds of error measures analysis, the MPE and the MAPE, and it was verified a good behaviour of the system.

\section{REFERENCES}

[1] Chun-yuan L.,Mian-huan W., Da-wei L.,"Combined control of Single Neuron PID and normal PID of switched reluctance motor," International Conference on Consumer electronics, Communications and Networks, CECNet 2011, pp 146-149, 16-18 Apr.2011.

[2] Lukic M., Emadi A.,"State Switching Control Technique for Switched Reluctance Motor Drives: Theory and implementation", IEEE Transactions on Industrial Electronics, Vol 57,Issue 9, pp 29322938,Sept. 2010.

[3] Muniraj, C., "Neural Network Based Speed Control for 6/4 Switched Reluctance Motor", International Conference on Computational Intelligence and Multimedia Applications, vol 1, pp 227-231, 13-15 Dec 2007.

[4] Lina L., Wu X., Yang Y., "Intelligent Control Algorithm Based on TS Fuzzy Neural Network PID and its Applications", International Conference on Intelligent Computation Technology and Automation, Vol 2, pp 553-557, 11-12 May, 2010.

[5] Maleki A.,Valianpour M., Mohammadi A., Darabi S., "Hybrid PIDlike fuzzy logic speed drive of switched reluctance motor", International Conference on Environement and Electrical Engineering , EEEIC 12, pp 794-797, 18-25 May 2012.

[6] Song A.,Cao Y.,Du D.,"Study of based Fuzzy-PID control for switched reluctance motor drive", International Conference on Computer Design and applications, ICCDA 2010, vol 3, pp 558-561, 25-27 Jun 2010.

[7] Rafael S, Pires A.J., Branco P.J.C., "An adaptive learning rate approach for an on-line neuro-fuzzy speed controller applied to a switched reluctance machine", Proceedings of the IEEE International Symposium on Industrial Electronics, ISIE 2005, vol 3, pp. 941-944, 20-23 Jun 2005.

[8] Hao Ying, "Design of a general class of Takagi-Sugeno fuzzy control systems", Proceedings of the American Control Conference, vol 6, pp 3746-3750, 4-6 Jun 1997.

[9] Baltazar Perreira, Silviano Rafael, Pires A.J., Branco P.J.C," Obtaining the Magnetic Characteristics of an 8/6-Switched Reluctance Machine: from FEM Analysis and Experimental Tests, IEEE Transaction on Industrial Electronics, Vol 52, $\mathrm{n}^{\circ}$ 6, Dec 2005, ISSN 0278-0046.

[10] P. Lobato, Silviano Rafael, A. J. Pires, "Magnetic Characteristics Model for an 8/6 Switched Reluctance Machine: Analytical Function Approach from Experimental Tests, International Conference on Computer as a Tool, EUROCON 2007, 9-12 Set. 2007, Varsóvia, Poland.

[11] Silviano Rafael, P.J. Costa Branco e A.J. Pires, "Position Control of an $8 / 6$ Switched Reluctance Machine without Current Sensor, 2nd International Conference on Power Engeneering, Energy and Electrical Drive, POWERENG 2009, 18-20 Mar. 2009, Lisbon, Portugal. ISBN 978-1-4244-2291-3. 